\title{
Surface flux evolution constraints for flux transport dynamos
}

\author{
R. H. Cameron ${ }^{1}$, D. Schmitt ${ }^{1}$, J. Jiang ${ }^{2}$, and E. Iş1k ${ }^{3}$ \\ 1 Max-Planck-Institut für Sonnensystemforschung, Max-Planck-Str. 2, 37191 Katlenburg-Lindau, Germany
e-mail: cameron@mps.mpg. de
2 Key Laboratory of Solar Activity, National Astronomical Observatories, Chinese Academy of Sciences, 100012 Beijing, PR China
3 Department of Physics, Faculty of Science \& Letters, Istanbul Kültür University, Ataköy Campus, 34156 Bakırköy, Istanbul, Turkey
}

Received 30 January 2012 / Accepted 3 May 2012

\begin{abstract}
The surface flux transport (SFT) model of solar magnetic fields involves empirically well-constrained velocity and magnetic fields. The basic evolution of the Sun's large-scale surface magnetic field is well described by this model. The azimuthally averaged evolution of the SFT model can be compared to the surface evolution of the flux transport dynamo (FTD), and the evolution of the SFT model can be used to constrain several near-surface properties of the FTD model.

We compared the results of the FTD model with different upper boundary conditions and diffusivity profiles against the results of the SFT model. Among the ingredients of the FTD model, downward pumping of magnetic flux, related to a positive diffusivity gradient, has a significant effect in slowing down the diffusive radial transport of magnetic flux through the solar surface. Provided the pumping was strong enough to give rise to a downflow of a magnetic Reynolds number of 5 in the near-surface boundary layer, the FTD using a vertical boundary condition matches the SFT model based on the average velocities above the boundary layer. The FTD model with a potential field was unable to match the SFT results.
\end{abstract}

Key words. dynamo - Sun: activity

\section{Introduction}

The flux transport dynamo (FTD) model attempts to explain the large-scale evolution of the Sun's magnetic field. The central ideas behind the model are that poloidal flux is wound up by differential rotation until it becomes sufficiently strong that magnetic buoyant flux tubes emerge through the solar surface. The erupted field is in the form of a bipolar active region, and the two opposite polarities are observed to be systematically tilted with respect to the equator (Joy's law). This tilt is such that the leading polarity is slightly closer to the equator than the following polarity. This latitudinal offset means that poloidal field has been created from the toroidal flux and, in the language of dynamo theory, the emergence process is a non-local alpha effect (Kitchatinov \& Olemskoy 2011a). The poloidal flux is then stretched and diffused by surface motions, reversing the polar fields and completing one half of a solar cycle. For a review of the basic ideas, see Charbonneau (2010). This picture has recently gained observational support from the analysis by Dasi-Espuig et al. (2010) and later by Kitchatinov \& Olemskoy (2011b), which show that the observed sunspot group tilt angles, which go into the construction of the poloidal source term, vary systematically from cycle to cycle in a way which possibly can explain the observed changes in cycle amplitudes during the twentieth century.

The winding up of the field by differential rotation and the rise of the tubes to the surface are hidden below the photosphere. The evolution of the field after it has broken through the surface can be and has been observed. The surface flux transport (SFT) model has been found to provide a good description of the large-scale evolution after emergence. For a detailed historical account, see Sheeley (2005). This model assumes that the magnetic field is purely radial at the surface and evolves passively driven by surface flows including differential rotation, meridional circulation and small-scale convective motions (granulation and supergranulation). The small-scale motions essentially cause the magnetic field to undergo a random walk and hence can be treated as a diffusive term. The ingredients which go into the SFT model are all observable, as is the output of the model - it is thus tightly constrained and supported by observations. For example, Cameron et al. (2010) showed that the SFT model, with the observed cycle-to-cycle variations of the tilt angle, can reproduce the inferred open magnetic flux of the Sun. Since the open flux during the maxima and minima of activity reflect the equatorial and axial dipole moments respectively, the model's ability to reproduce the open flux over an extended period is a strong test of the model.

In this paper we investigate what constraints can be inferred for the FTD model, given that it should also reproduce the same surface dynamics as is described by the SFT model. We have used the FTD code developed at the Max-Planck-Institut für Sonnensystemforschung. For the SFT model we have used a 1-D surface flux transport model developed at the MPS. The 1-D SFT model includes exactly the component which can be compared between the two models. The details of the two approaches will be discussed in Sect. 2. In Sect. 3 we present the results of the simulations and compare the two models. The effect of varying some of the most important unconstrained parameters and the boundary condition will be discussed in Sect. 4 . We will conclude in Sect. 5 with the finding that the appropriate boundary condition for FTD models is that the field is vertical at the surface, and that a certain amount of turbulent pumping must be included for the FTD simulations to mimic the surface behavior of the SFT model and to thus match the observations.

Downward pumping has in particular been discussed for the base of the solar convection zone. Direct numerical simulations 
show a downward transport of large-scale magnetic field near the base of convective unstable layers (e.g., Jennings et al. 1992; Tobias et al. 1998, 2001; Ossendrijver et al. 2002) though it is not clear whether this should be interpreted in terms of turbulent pumping (Zeldovich 1957; Rädler 1968) or of topological pumping (Drobyshevski \& Yuferev 1974). In mean-field dynamo models of the solar cycle turbulent pumping is often not included. In those cases where it is included, most of the attention is focussed on its role in transporting flux into the top of the stable layer immediately below the convection zone (see for example Brandenburg et al. 1992; Käpylä et al. 2006; Do Cao \& Brun 2011; Kitchatinov \& Olemskoy 2011a, 2012). The effect of pumping throughout the convection zone was considered by Guerrero \& de Gouveia Dal Pino (2008), who found that the pumping affects whether the preferred mode of the solution is dipolar or quadrupolar, and identified the possible importance of radial transport by the pumping in the dynamo process. In this paper we are especially paying attention to the pumping in the near-surface boundary layer (Miesch \& Hindman 2011) which is necessary to match FTD to SFT simulations of the magnetic flux on the solar surface.

\section{Physical models and numerical codes}

\subsection{The flux transport dynamo (FTD) model}

The flux transport dynamo equations describe the induction, advection and diffusion of a large-scale magnetic field. Their axisymmetric form is:

$$
\begin{aligned}
\frac{\partial A}{\partial t}= & \eta(r)\left(\nabla^{2}-\frac{1}{(r \sin \theta)^{2}}\right) A \\
& -\frac{\boldsymbol{u}_{\mathrm{m}}(r, \theta)+\boldsymbol{u}_{\mathrm{p}}(r, \theta)}{r \sin \theta} \cdot \nabla(A r \sin \theta)+\alpha(B) \\
\frac{\partial B}{\partial t}= & \eta(r)\left(\nabla^{2}-\frac{1}{(r \sin \theta)^{2}}\right) B+\frac{1}{r} \frac{\partial \eta}{\partial r} \frac{\partial r B}{\partial r} \\
& -r \sin \theta\left(\boldsymbol{u}_{\mathrm{m}}(r, \theta)+\boldsymbol{u}_{\mathrm{p}}(r, \theta)\right) \cdot \nabla\left(\frac{B}{r \sin \theta}\right) \\
& -B \nabla \cdot\left(\boldsymbol{u}_{\mathrm{m}}(r, \theta)+\boldsymbol{u}_{\mathrm{p}}(r, \theta)\right) \\
& +r \sin \theta\left(\nabla \times\left(A \hat{\boldsymbol{e}}_{\phi}\right)\right) \cdot \nabla \Omega(r, \theta)
\end{aligned}
$$

where $A(r, \theta)$ is the $\phi$-component of the vector potential associated with the poloidal components of $\boldsymbol{B}, B(r, \theta)$ is the toroidal component of the field, $\boldsymbol{u}_{\mathrm{m}}(r, \theta)$ is the velocity in the meridional plane, $\Omega(r, \theta)$ is the angular velocity, $\boldsymbol{u}_{\mathrm{p}}(r, \theta)$ is a velocity field corresponding to the pumping of the magnetic field and $\alpha$ is a source term in the equation for $A$ corresponding to the generation of poloidal flux from toroidal flux. Since the purpose of the current study is to compare the response of the SFT and FTD models to equivalent sources of poloidal flux, we restrict ourselves to the case $\alpha=0-$ for other choices of $\alpha$ we would need to modify the source term in the SFT model accordingly. In relation to the term $\boldsymbol{u}_{\mathrm{p}}$ it is important to note that, as in Guerrero $\&$ de Gouveia Dal Pino (2008), it does not correspond to a true motion of the fluid and need not satisfy $\nabla \cdot \rho \boldsymbol{u}_{\mathrm{p}}=0$. Rather it is a parametrization of the effect of the turbulent motions on the field: for diamagnetic pumping it has the form $\boldsymbol{u}_{\mathrm{p}}(r, \theta)=-\frac{1}{2} \nabla \eta$. Other effects, such as topological pumping, are also expected to transport the field downwards, and for this study we assume that the combined effects of the turbulent convection, including diamagnetic pumping, can be written in the form $\boldsymbol{u}_{\mathrm{p}}(r, \theta)=-\frac{k}{2} \nabla \eta$, with $k \geq 1$. This choice allows us to vary the magnitude of the pumping in the near surface layers.
We solve the dynamo Eqs. (1) and (2) forward in time in a spherical shell $r_{0} \leq r \leq R_{\odot}$ with inner boundary $r_{0}=0.65 R_{\odot}$ matching to a perfect conductor and outer boundary matching to either a radial field or vacuum conditions outside. This leads to the boundary conditions

$A=0 \quad$ and $\quad \frac{\partial}{\partial r}(r B)=0 \quad$ at $\quad r=r_{0}$

and

$\frac{\partial}{\partial r}(r A)=0 \quad$ and $\quad B=0 \quad$ at $\quad r=R_{\odot}$

for the field to be vertical at the Sun's surface, or alternatively

$$
\begin{aligned}
A & =\sum_{k} a_{k} P_{k}^{1}(\cos \theta) \\
\frac{\partial A}{\partial r} & =-\sum_{k}(k+1) a_{k} P_{k}^{1}(\cos \theta) \quad \text { and } \\
B & =0 \quad \text { at } \quad r=R_{\odot}
\end{aligned}
$$

for matching to a potential field outside. At the poles we require regularity resulting in

$A=B=0 \quad$ at $\quad \theta=0, \pi$.

The equations are discretized using second order accurate centered finite differences on an equidistant grid and forwarded in time with an Alternating Direction Implicit scheme for the diffusion terms and an explicit scheme for the induction and advection terms. The code is tested against the dynamo benchmark of Jouve et al. (2008).

For current purposes we will consider $\alpha=0$ so that there is no source of poloidal field during the simulation. From any initial condition the field must then eventually decay towards zero, however at any finite time the magnetic field will depend on the initial field and can be compared with the result of the SFT model.

For the initial condition we take

$$
\begin{aligned}
A=\frac{1}{8} R_{\odot}(1 & \left.+\operatorname{erf}\left(\frac{r-r_{1}}{\Delta r}\right)\right) \times\left(1+\operatorname{erf}\left(\frac{\theta-\theta_{1}}{\Delta \theta}\right)\right) \\
& \times\left(1-\operatorname{erf}\left(\frac{\theta-\theta_{2}}{\Delta \theta}\right)\right),
\end{aligned}
$$

$B=0$,

where $r_{1}=0.80 R_{\odot}, \theta_{1}=80^{\circ}, \theta_{2}=86^{\circ}, \Delta \theta=2.9^{\circ}$ and $\Delta r=0.01 R_{\odot}$. This corresponds to an isolated bipole emerging on the solar surface slightly north of the equator. Since both the SFT and FTD studied here are linear, the evolution of such a bipole is independent of the emergence and evolution of other emerging groups. To check that the models are consistent, it is thus sufficient to follow the evolution of a single feature starting near the equator to the poles.

The velocity in the meridional plane is taken from Dikpati et al. (2004). The velocity components can be written in terms of a stream function as

$\boldsymbol{u}_{\mathrm{m}}(r, \theta)=\frac{v_{0}}{\rho} \frac{1}{r \sin \theta} \frac{\partial \Psi \sin \theta}{\partial \theta} \hat{\boldsymbol{e}}_{r}-\frac{v_{0}}{r \rho} \frac{\partial r \Psi}{\partial r} \hat{\boldsymbol{e}}_{\theta}$,

where

$\xi=\frac{R_{\odot}}{r}-0.985$,

$\rho=\xi^{m}$ 
and

$$
\begin{aligned}
\Psi(r, \theta)= & \frac{R_{\odot}}{r} \\
& \times\left(\frac{-1}{m+1} \xi^{m+1}+\frac{c_{1}}{2 m+1} \xi^{2 m+1}-\frac{c_{2}}{2 m+p+1} \xi^{2 m+p+1}\right) \\
& \times \sin ^{q+1} \theta \cos \theta
\end{aligned}
$$

with

$c_{1}=\frac{(2 m+1)(m+p)}{(m+1) p} \xi_{0}^{-m}$,

$c_{2}=\frac{(2 m+p+1) m}{(m+1) p} \xi_{0}^{-(m+p)}$.

For the reference case we take $q=1.5, m=1.5, p=3, r_{0}=0.7$ and $\xi_{0}=\xi\left(r_{0}\right)$. In all cases $v_{0}$ is chosen so that the maximum meridional velocity at $r=R_{\odot}$ is $15 \mathrm{~m} / \mathrm{s}$. The resulting velocity approximates the meridional circulation in the solar convection zone derived by numerical modeling of Rempel (2005) and Kitchatinov \& Rüdiger (2005) and is consistent with the velocity in the subsurface layers as derived from helioseismology (as measured, e.g. by Giles et al. 1997).

The differential rotation is taken from Belvedere et al. (2000), and is also used e.g. by Kitchatinov \& Olemskoy (2011b),

$\Omega(r, \theta)=\sum_{j=0}^{2} \cos \left(2 j\left(\frac{\pi}{2}-\theta\right)\right) \sum_{i=0}^{4} c_{i j} r^{i}$

where the coefficients $c_{i j}$ are given in Table 1 of Belvedere et al. (2000). This approximates the internal rotation of the Sun as derived from helioseismological inversions (as reported, e.g., by Schou et al. 1998).

For the diffusivity we assumed

$$
\begin{aligned}
\eta(r)= & \eta_{0}+\frac{\eta_{1}-\eta_{0}}{2}\left(1+\operatorname{erf}\left(\frac{r-0.7 R_{\odot}}{0.02 R_{\odot}}\right)\right) \\
& +\frac{\eta_{2}-\eta_{1}}{2}\left(1+\operatorname{erf}\left(\frac{r-0.95 R_{\odot}}{0.02 R_{\odot}}\right)\right)
\end{aligned}
$$

with $\eta_{0}=0.1 \mathrm{~km}^{2} \mathrm{~s}^{-1}, \eta_{1}=10 \mathrm{~km}^{2} \mathrm{~s}^{-1}$ and $\eta_{2}=250 \mathrm{~km}^{2} \mathrm{~s}^{-1}$, see e.g. Muñoz-Jaramillo et al. (2011). Here $\eta_{2}$ represents the turbulent diffusivity in the near-surface layers, $\eta_{1}$ in the bulk of the convection zone, and $\eta_{0}$ in the overshoot region at the base of the convection zone. Other choices will be considered in Sect. 4.

Recently Kitchatinov \& Olemskoy (2011a) have highlighted the importance of downward pumping of magnetic fields due to gradients in the turbulent diffusivity, and have argued that this is particularly important near the base of the convection zone. We here consider downward pumping in the near-surface layers. We have found it necessary to increase the strength of the downward pumping from its usual value of $(1 / 2)(\partial \eta / \partial r)$ in order to obtain a match between the FTD and SFT models. We have therefore introduced in Eqs. (1) and (2) a scaling factor $k$ which we have varied between 0 and 20 . The diffusivity profile and the corresponding diamagnetic pumping velocity with $k=1$ is shown in Fig. 1.

While we solve both Eqs. (1) and (2) we note that the comparison with the SFT model only depends on Eq. (1) as $\alpha=0$. The physical ingredients which affect $A$ are the meridional flow, the radial and latitudinal diffusion, and the downward pumping.

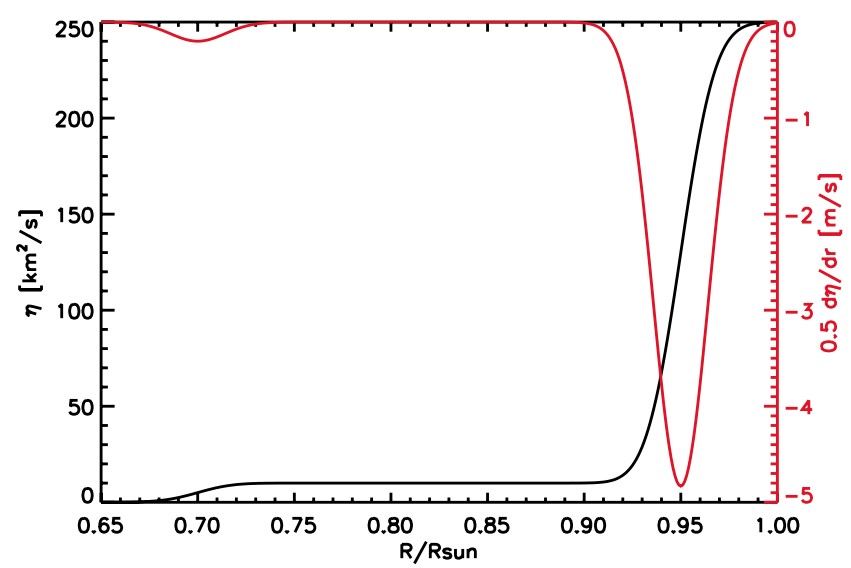

Fig. 1. The assumed profile of the turbulent diffusivity is shown in black, the effective radial velocity due to the radial derivative of the turbulent diffusivity, for the case $k=1$, is shown in red.

\subsection{The surface flux transport (SFT) model}

The SFT model, which describes the evolution of the magnetic field on the solar surface, assumes that the field is vertical and evolves passively under the action of the surface flows. The surface differential rotation and surface meridional flow towards the poles are modeled as systematic flows, while granular and supergranular flows are assumed to only cause the fields to diffuse across the solar surface. In this sense correlations between the radial component of the magnetic field $B_{r}$ and the supergranular velocity field $U_{\mathrm{SG}}$ are ignored, i.e. it is assumed that $\left\langle U_{\mathrm{SG}} B_{r}\right\rangle=\left\langle U_{\mathrm{SG}}\right\rangle\left\langle B_{r}\right\rangle$, and since differential rotation and the meridional flow have been removed, $\left\langle U_{\mathrm{SG}}\right\rangle=0$. This assumption is not justified since the magnetic field and supergranular velocity fields are correlated, as the magnetic field is located at the edge of the supergranules. This presumably accounts for the observation by Meunier (2005) that magnetic fields rotate faster than the local plasma, with the extent of the prograde motion depending on the technique used to measure the velocity. In the current context this is a small effect which can be ignored.

The SFT model additionally assumes that there is no transport of flux, either advective or diffusive, across the solar surface. The relevant equation is

$$
\begin{aligned}
\frac{\partial B_{r}}{\partial t}= & -\omega(\theta) \frac{\partial B_{r}}{\partial \phi}-\frac{1}{R_{\odot} \sin \theta} \frac{\partial}{\partial \theta}\left[v(\theta) B_{r} \sin \theta\right] \\
& +\frac{\eta}{R_{\odot}^{2}}\left[\frac{1}{\sin \theta} \frac{\partial}{\partial \theta}\left(\sin \theta \frac{\partial B_{r}}{\partial \theta}\right)+\frac{1}{\sin ^{2} \theta} \frac{\partial^{2} B_{r}}{\partial \phi^{2}}\right]
\end{aligned}
$$

where $B_{r}$ is the radial component of the magnetic field, $\theta$ is the heliographic colatitude, and $\phi$ is the heliographic longitude. $\omega(\theta)$ is the surface differential rotation and $v(\theta)$ is the surface meridional flow. For the purposes of comparison with the FTD simulation, we take $v(\theta)=\boldsymbol{u}_{\mathrm{m}}\left(R_{\odot}, \theta\right) \cdot \hat{\boldsymbol{e}}_{\theta}, \omega(\theta)=\Omega\left(R_{\odot}, \theta\right)$, and $\eta=\eta\left(R_{\odot}\right)=250 \mathrm{~km}^{2} \mathrm{~s}^{-1}$.

For comparison with the FTD simulation, we can only use the azimuthally averaged (signed) field strength. This averaged field is independent of the initial structure of the field in the azimuthal direction and hence we can take

$B_{r}(\theta)=\frac{1}{r \sin \theta} \frac{\partial}{\partial \theta}(A \sin \theta)$

as our initial condition, consistent with the initial condition of the FTD simulation. The solution to this one dimensional problem, $B_{r}(\theta, t)$, can be directly compared to $R_{\odot}(\partial / \partial \theta)(A \sin \theta)$ 


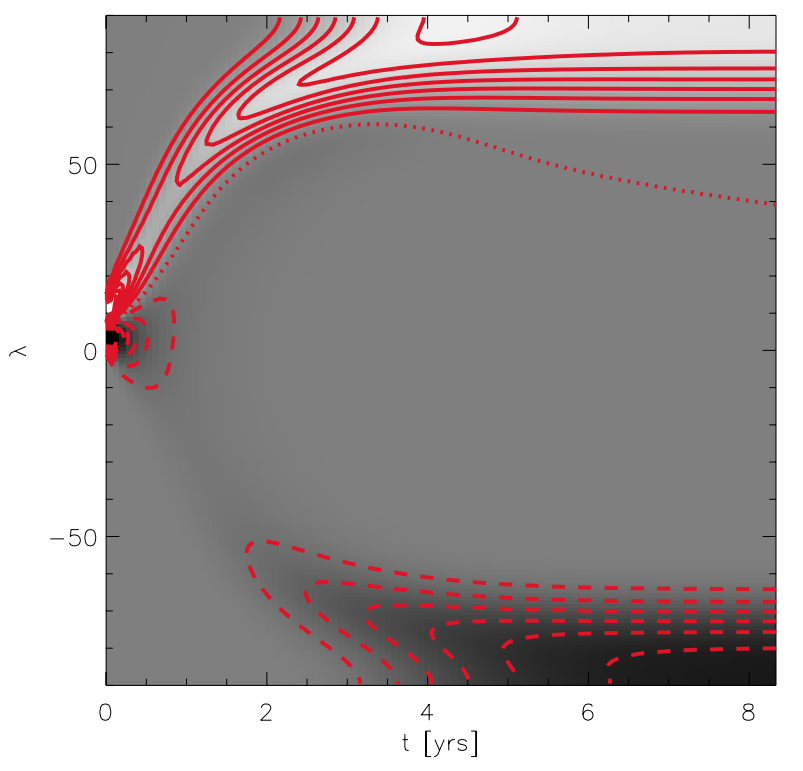

Fig. 2. Evolution of the azimuthally averaged signed field strength from the SFT simulation, with black and white representing opposite polarities saturated at $36 \%$ of the initial azimuthally averaged field strength. The solid and dashed red contours indicate where the field strength reaches $\pm 5 \%, \pm 10 \%$, etc. of its maximum value, with the dotted curve representing the 0 level.

from the FTD simulation. We have used the code described in Cameron \& Schüssler (2007) to solve this 1-D surface flux transport problem.

\section{Reference case}

In Fig. 2 the evolution of the surface flux according to the SFT model is displayed. Figure 3 shows the surface latitudinal dependence of the different FTD models with the vertical boundary condition with that from the SFT model shown for comparison. We note that for $k \gtrsim 5$, both FTD and SFT models match very well. For $k=0$ the match is much worse, e.g. there is too little flux in the southern hemisphere $(\theta>90)$ at $t=72$ months. In the northern hemisphere at $t=18$ months, the amplitude of the field in the FTD model is greater for both polarities. By $t=72$ months the amplitude of the field in the southern hemisphere has also fallen as the opposite polarities are merging. This implies that downward pumping corresponding to at least $k=5$ is required for the FTD model to be consistent with the SFT model and therefore with observations.

The reason why downward pumping is important can be seen in Fig. 4 where, in the case without pumping, the diffusive emergence of flux through the upper boundary is obvious. This emergence of flux is strongly inhibited by the downward pumping. The requirement that the downward pumping should inhibit the diffusion of flux across the surface is captured by the corresponding magnetic Reynolds number $R_{\mathrm{m}}$ being larger than 1:

$$
\begin{aligned}
R_{\mathrm{m}} & =\frac{\left|\boldsymbol{u}_{\mathrm{p}}\right| L}{\eta} \\
& =\frac{(k / 2)(\partial \eta / \partial r) L}{\eta} \\
& \approx \frac{(k / 2)\left[\left(\eta_{2}-\eta_{1}\right) / L\right] L}{\left(\eta_{2}+\eta_{1}\right) / 2} \\
& \approx k
\end{aligned}
$$

for $\eta_{1} \ll \eta_{2}$.
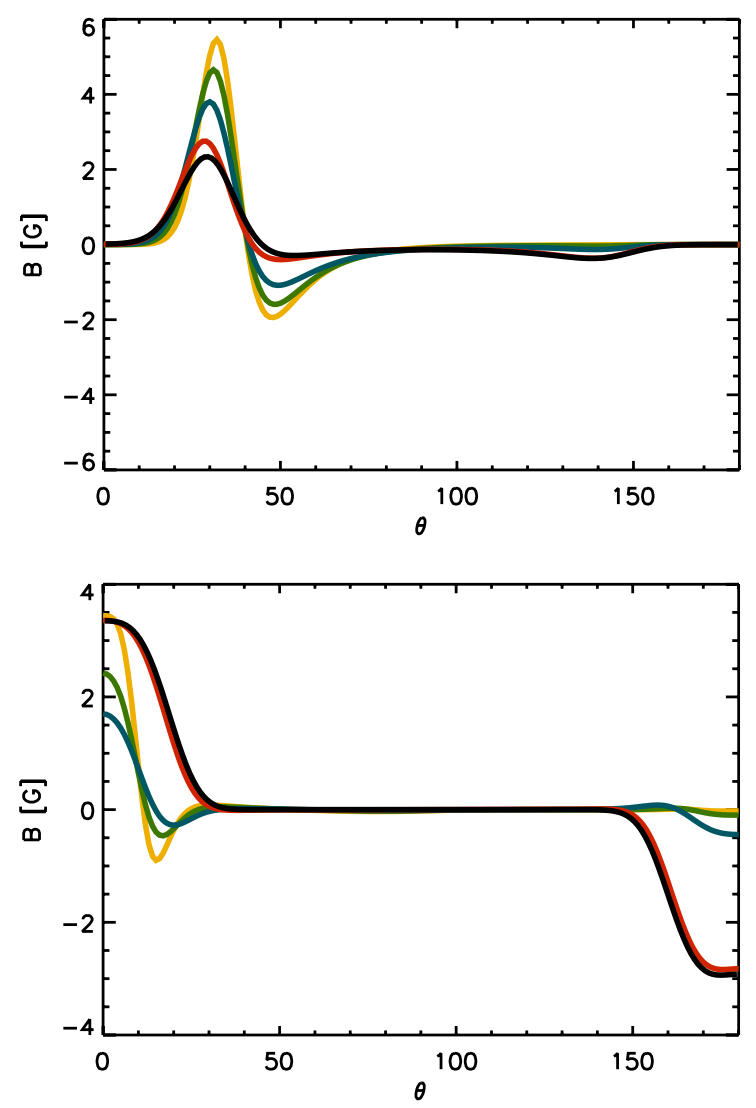

Fig. 3. Azimuthally averaged signed field from the SFT model (black) vs. surface field from FTD simulations at $t=18$ months (top) and $t=$ 72 months (bottom) with a vertical field outer boundary and pumping with factors $k=5$ (red), 2 (blue), 1 (green), and 0 (yellow).

Here $\boldsymbol{u}_{\mathrm{p}}$ is the pumping velocity and $L$ is the boundary layer thickness corresponding to the region over which $\eta$ changes from its value throughout the bulk of the convection zone $\eta_{1}$ to its surface values $\eta_{2}$. Basing $R_{\mathrm{m}}$ on the mean over this transition yields $R_{\mathrm{m}} \approx k$ when $\eta_{1} \ll \eta_{2}$. To prevent diffusive transport, we require $R_{\mathrm{m}} \gg 1$, which for our purposes appears to be achieved by $R_{\mathrm{m}} \approx k \gtrsim 5$. This argument also shows that, for the chosen diffusivity profile, the downward pumping velocity needs to be of the order of $25 \mathrm{~m} / \mathrm{s}$. In reality, this pumping can be due to a mixture of turbulent and topological effects and the choice of the form for $\boldsymbol{u}_{\mathrm{p}}$ is not critical.

\section{Effects of varying the diffusivity and meridional velocity}

In this section we briefly discuss four variations to the above reference case. Explicitly, we consider one simulation with a potential field boundary condition, one with a different diffusivity profile, one with anisotropic diffusivity, and one with a different meridional flow profile.

\subsection{Potential field boundary condition}

Figure 5 shows the evolution of the field from the FTD simulations with a potential field boundary condition. The SFT result is again shown for reference. With this boundary condition we see that the match is always poor. This is because there is now a strong diffusive flux across the solar surface, corresponding to the retraction of field lines, as can be seen in Fig. 6. Hence for the 

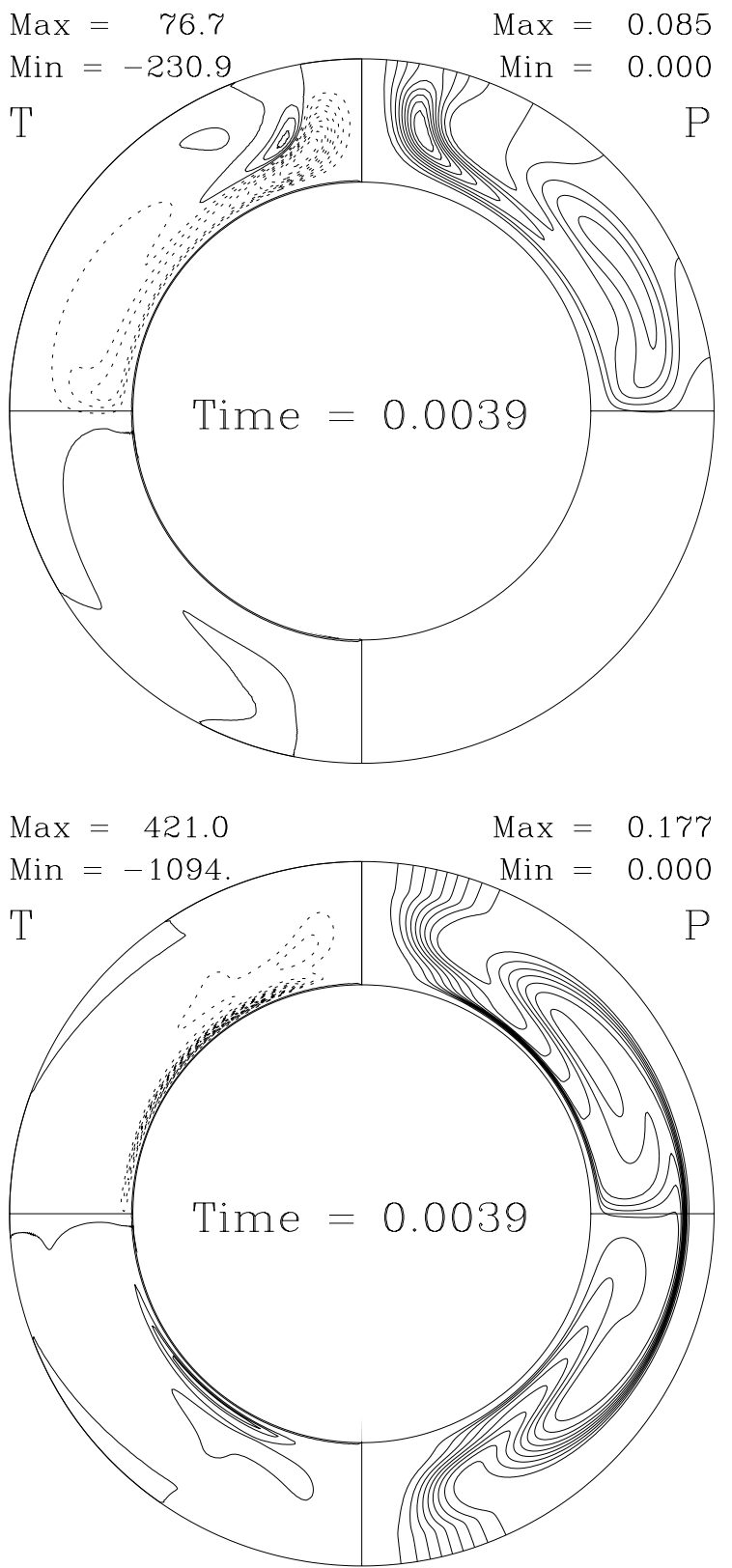

Fig. 4. Magnetic field structure from the FTD simulations at $t=$ 72 months for the case with a vertical boundary condition and $k=0$ (top) and $k=5$ (bottom). In each subpanel the left half shows contours of the toroidal field $(T)$, the right panel shows selected fieldlines of the poloidal field (formally it shows contours of $P=r \sin \theta A(r, \theta)$ ). The dashed contours of the toroial field indicate negative fields, the solid contours represent either zero or positive toroidal field. In particular the solid contours which touch the boundaries correspond to zero toroidal flux.

FTD to be consistent with the SFT model, we need strong downward pumping $(k \gtrsim 5)$ and a vertical boundary condition. These two requirements correspond directly to the assumptions of the SFT model, that the only sources are those which are explicitly put in (i.e. no diffusive sources) and that the field at the surface is vertical. We note that extensions to the SFT model have slightly relaxed the assumption that there are no diffusive fluxes (see for example Baumann et al. 2006), but the values of the radial diffusivities suggested there correspond to long decay times of the SFT fields at the poles which are still not comparable to our FTD simulations with $k=0,1$ or 2 .
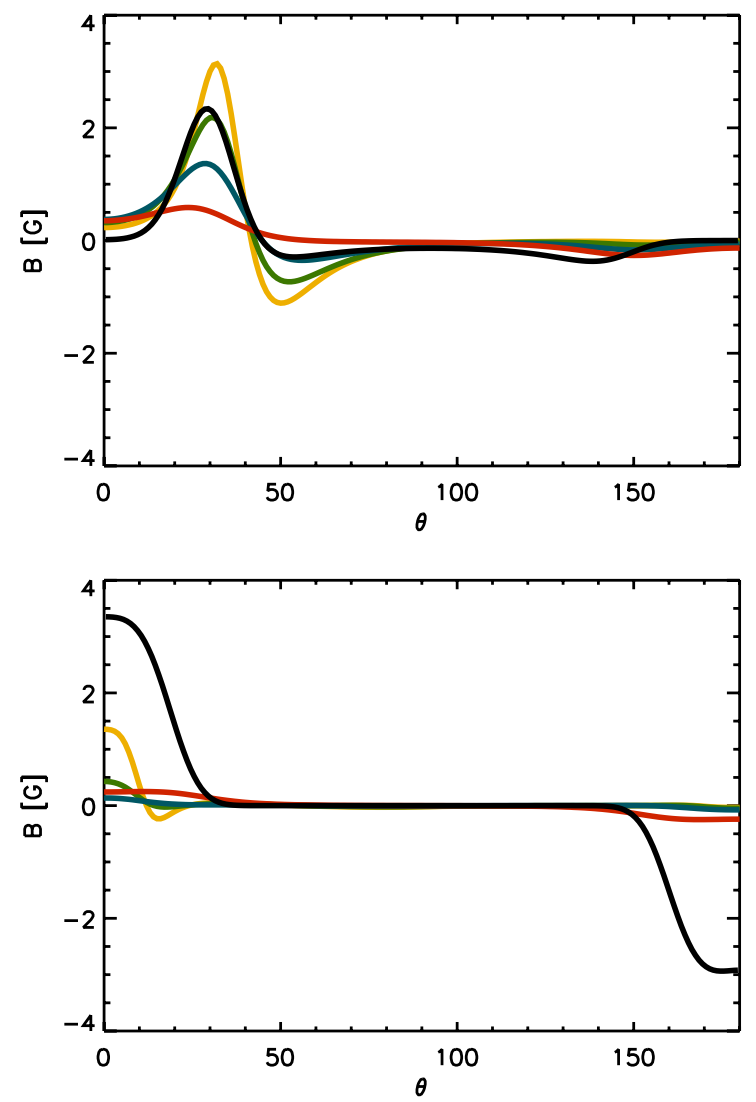

Fig. 5. Similar to Fig. 3 except a potential field upper boundary condition was used for the FTD simulations. The black line shows the surface field from the SFT model, the colored lines show the FTD results for different values of $k$.

\subsection{High diffusivity in the bulk of the convection zone}

For the simulation with a different $\eta$, we considered $\eta_{0}=$ $0.1 \mathrm{~km}^{2} \mathrm{~s}^{-1}, \eta_{1}=100 \mathrm{~km}^{2} \mathrm{~s}^{-1}$ and $\eta_{2}=250 \mathrm{~km}^{2} \mathrm{~s}^{-1}$ in Eq. (15). This is similar to the diffusivity profile of the reference case discussed in Sect. 3 except that the diffusivity in the bulk of the convection zone has been raised to $100 \mathrm{~km}^{2} \mathrm{~s}^{-1}$. The average magnetic diffusivity of the transition between low and high velocities is then higher, the velocity by contrast has fallen. The magnetic Reynolds number is then $R_{\mathrm{m}}=(1.5 k) / 3.5$. To have $R_{\mathrm{m}} \gtrsim 5$ we then need $k \gtrsim 5 \times(3.5 / 1.5) \approx 12$; and indeed we found that with $k=10$ the FTD and SFT models were close to, though not quite, matching.

\subsection{Anisotropic diffusivity}

In our third experiment, we studied the effect of an anisotropy in the diffusivity near the surface. We used the same formula for the different components of $\eta$ (i.e., Eq. (18)), but with different values of the surface diffusivity, $\eta_{2}$, for the horizontal and vertical directions. Motivated by the work of Miesch \& Hindman (2011), we chose the longitudinal and latitudinal diffusivities to be the same, $\eta_{2}=250 \mathrm{~km}^{2} \mathrm{~s}^{-1}$, and the radial diffusivity to be an order of magnitude smaller, $\eta_{2}=25 \mathrm{~km}^{2} \mathrm{~s}^{-1}$. We based the downward pumping, $\boldsymbol{u}_{\mathrm{p}}$, on the gradient of the vertical component of the diffusivity. The comparison of the FTD and SFT models, for several values of $k$, are shown in Fig. 7 for two times. Importantly, a strong downward pumping with $k>10$ is needed for the FTD to match the SFT surface evolution. 

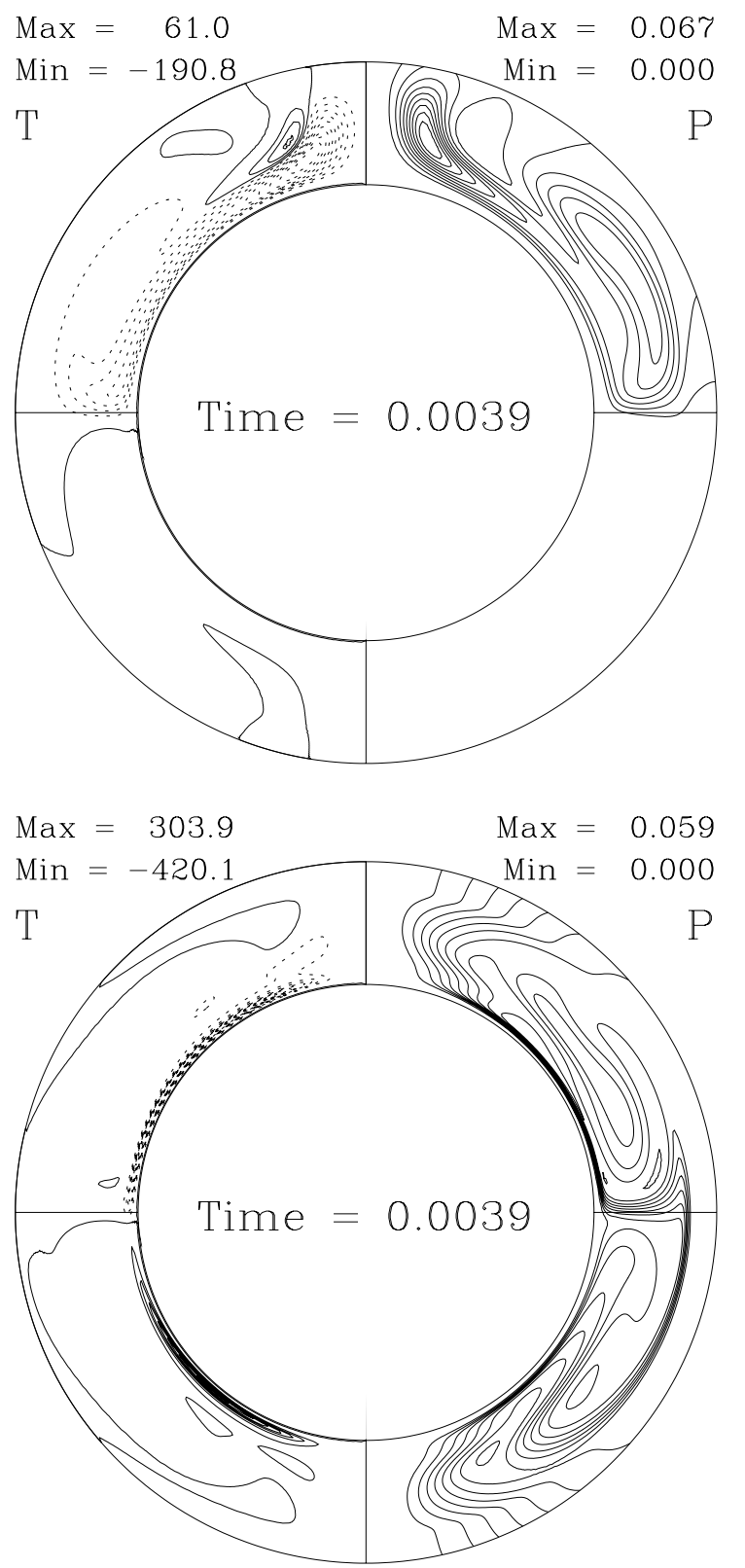

Fig. 6. The magnetic field structure from the FTD model in the same format as in Fig. 4 when the potential field boundary condition is used.

\subsection{Variation of the meridional circulation}

For the simulation with a different meridional velocity profile, we used the same form as described in Eqs. (11) to (16) but with $p=0.25, q=0$ and $m=0.5$ (Dikpati \& Charbonneau 1999). For this choice of the meridional flow, the FTD and SFT models always evolve differently, even though the surface velocity is used for the SFT calculation (Fig. 8, top). The reason is that the meridional velocity in this case is not constant above the transition from low to high diffusivities, which occurs at about $0.95 R_{\odot}$. The magnetic field in the FTD calculation sees a range of velocities above the "boundary layer" associated with the transition and the strong pumping. Because the diffusivity is reasonably large above the transition, the magnetic flux should essentially be advected according to the average meridional flow in this layer. Therefore the surface field is effectively advected with the average meridional flow speed in the boundary layer, and not with its surface value. This indeed happens as can be seen in
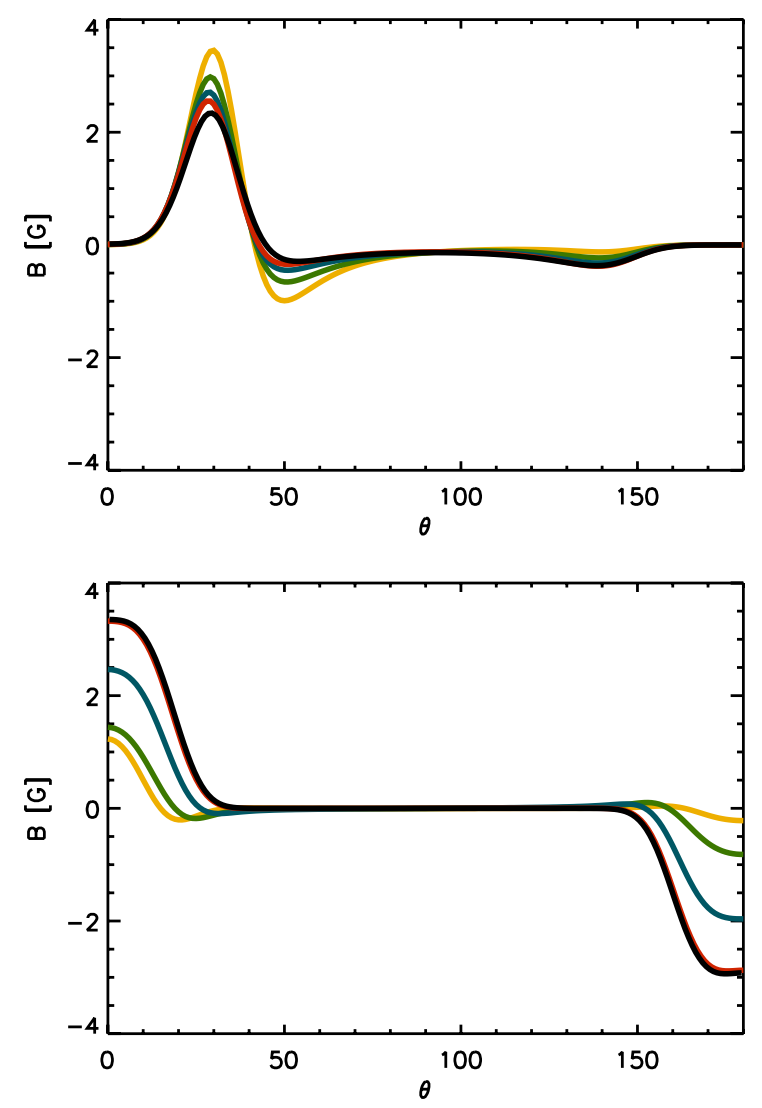

Fig. 7. The surface field from the reference SFT simulations at $t=$ 18 months (top) and $t=72$ months (bottom) are shown in black. The results of the FTD simulation with a vertical field outer boundary and an anisotropic near-surface diffusivity $\left(250 \mathrm{~km}^{2} \mathrm{~s}^{-1}\right.$ in the horizontal directions and $25 \mathrm{~km}^{2} \mathrm{~s}^{-1}$ in the vertical direction). The vertical pumping is based on the vertical diffusivity gradient with $k=20$ (red), 10 (blue), 5 (green), and 0 (yellow).

Fig. 8 (bottom). It is noteworthy that this mainly affects the time it takes for the flux to reach the poles, not the amount that eventually gets there. The meridional flow is difficult to measure at depths below about $10 \mathrm{Mm}$; in the top $10 \mathrm{Mm}$ the indications from helioseismology are that the meridional flow first increases and then decreases (Basu \& Antia 2010).

There is also an observed near-surface shear in the differential rotation (Thomson et al. 1995), which has been used to explain the observed difference between the rotation of magnetic features (Snodgrass 1983) and the rate deduced from surface Doppler observations of the flow. For a review of the observational results, see Beck (2000). The conventional explanation is made in terms of the "anchoring depth" of the features (Nesme-Ribes et al. 1993). Our suggestion is that the observed rotation rate of magnetic features is partly due to the average value in a high-diffusivity layer, which is partially isolated from the deeper dynamics by a boundary layer associated with magnetic pumping.

\section{Conclusion}

With a vertical outer boundary condition and enough pumping the FTD model is consistent with the SFT model. The pumping needs to be strong enough to result in a magnetic Reynolds number of approximately 5 . With a potential boundary condition or weaker pumping, the models do not match. This strong pumping requires a velocity which is greater than the standard value 
R. H. Cameron et al.: Surface flux evolution constraints for flux transport dynamos
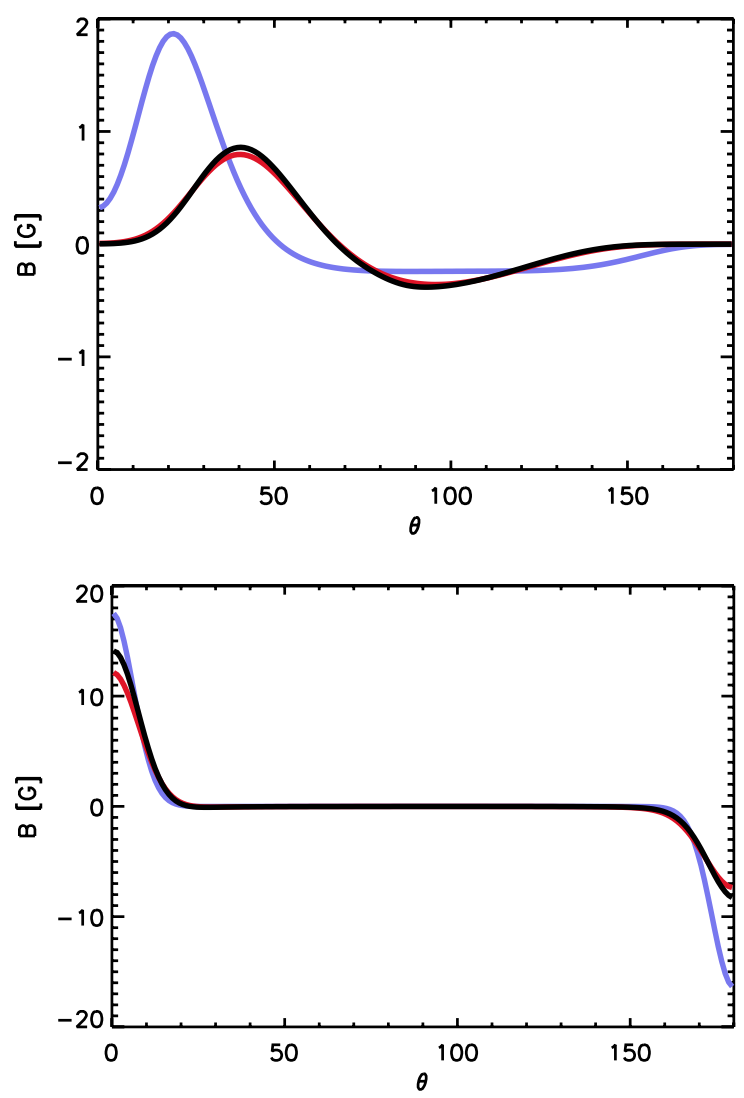

Fig. 8. The surface field from FTD simulations at $t=18$ months (top) and $t=72$ months (bottom) with a vertical field outer boundary using the $k=5$ and the meridional velocity profile with $p=0.25, q=0$ and $m=0.5$ (black) is used. In this case there is a strong near-surface shear. The results from the SFT model using the surface meridional velocity (blue) and using the average of the meridional velocity above $0.95 R_{\odot}$ (red) are shown for comparison.

given by mean-field theory for diamagnetic pumping. Since the SFT model matches observations, it follows that the vertical field boundary condition and sufficient downward pumping are required for the FTD model to match the observed surface evolution of the field.
Acknowledgements. The authors gratefully acknowledge Manfred Schüssler for enlightening discussions on various aspects of this paper. J.J. acknowledges financial support from the National Natural Science Foundations of China through grants 11173033, 11178005, 11125314 and a Young Researcher Grant of the National Astronomical Observatories, Chinese Academy of Sciences.

\section{References}

Basu, S., \& Antia, H. M. 2010, ApJ, 717, 488

Baumann, I., Schmitt, D., \& Schüssler, M. 2006, A\&A, 446, 307

Beck, J. G. 2000, Sol. Phys., 191, 47

Belvedere, G., Kuzanyan, K. M., \& Sokoloff, D. 2000, MNRAS, 315, 778

Brandenburg, A., Moss, D., \& Tuominen, I. 1992, in The Solar Cycle, ed. K. L. Harvey, ASP Conf. Ser., 27, 536

Cameron, R., \& Schüssler, M. 2007, ApJ, 659, 801

Cameron, R. H., Jiang, J., Schmitt, D., \& Schüssler, M. 2010, ApJ, 719, 264

Charbonneau, P. 2010, Liv. Rev. Sol. Phys., 7, 3

Dasi-Espuig, M., Solanki, S. K., Krivova, N. A., Cameron, R., \& Peñuela, T. 2010, A\&A, 518, A7

Dikpati, M., \& Charbonneau, P. 1999, ApJ, 518, 508

Dikpati, M., de Toma, G., Gilman, P. A., Arge, C. N., \& White, O. R. 2004, ApJ, 601,1136

Do Cao, O., \& Brun, A. S. 2011, Astron. Nachr., 332, 907

Drobyshevski, E. M., \& Yuferev, V. S. 1974, J. Fluid Mech., 65, 33

Giles, P. M., Duvall, T. L., Scherrer, P. H., \& Bogart, R. S. 1997, Nature, 390, 52 Guerrero, G., \& de Gouveia Dal Pino, E. M. 2008, A\&A, 485, 267

Jennings, R. L., Brandenburg, A., Nordlund, A., \& Stein, R. F. 1992, MNRAS, 259,465

Jouve, L., Brun, A. S., Arlt, R., et al. 2008, A\&A, 483, 949

Käpylä, P. J., Korpi, M. J., \& Tuominen, I. 2006, Astron. Nachr., 327, 884

Kitchatinov, L. L., \& Olemskoy, S. V. 2011a, Astron. Nachr., 332, 496

Kitchatinov, L. L., \& Olemskoy, S. V. 2011b, Astron. Lett., 37, 656

Kitchatinov, L. L., \& Olemskoy, S. V. 2012, Sol. Phys., 276, 3

Kitchatinov, L. L., \& Rüdiger, G. 2005, Astron. Nachr., 326, 379

Meunier, N. 2005, A\&A, 436, 1075

Miesch, M. S., \& Hindman, B. W. 2011, ApJ, 743, 79

Muñoz-Jaramillo, A., Nandy, D., \& Martens, P. C. H. 2011, ApJ, 727, L23

Nesme-Ribes, E., Ferreira, E. N., \& Mein, P. 1993, A\&A, 274, 563

Ossendrijver, M., Stix, M., Brandenburg, A., \& Rüdiger, G. 2002, A\&A, 394, 735

Rädler, K.-H. 1968, Z. Naturf. A, 23, 1851

Rempel, M. 2005, ApJ, 622, 1320

Schou, J., Antia, H. M., Basu, S., et al. 1998, ApJ, 505, 390

Sheeley, Jr., N. R. 2005, Liv. Rev. Sol. Phys., 2, 5

Snodgrass, H. B. 1983, ApJ, 270, 288

Thomson, D. J., Maclennan, C. G., \& Lanzerotti, L. J. 1995, Nature, 376, 139

Tobias, S. M., Brummell, N. H., Clune, T. L., \& Toomre, J. 1998, ApJ, 502, L177

Tobias, S. M., Brummell, N. H., Clune, T. L., \& Toomre, J. 2001, ApJ, 549, 1183

Zeldovich, Y. B. 1957, JETP, 4, 460 